14

\title{
Propagation of HF radio waves over northerly paths: measurements, simulation and systems aspects
}

\author{
E. MICHAEL WARRINGTON, ALAN J. STOCKER, NIKOLAY Y. ZAALOV, \\ DAVID R. SIDDLE and IGOR A. NASYROV
}

Department of Engineering, University of Leicester, U.K.

Large deviations in the direction of arrival of ionospherically propagating radio signals from the Great Circle Path (GCP) have serious implications for the planning and operation of communications and radiolocation systems operating within the HF-band. Very large deviations are particularly prevalent in the polar and sub-auroral regions where signals often arrive at the receiver with bearings displaced from the great circle direction by up to $\pm 100^{\circ}$ or more. Measurements made over several paths are presented in this paper, and the principle causes of off-great circle propagation outlined. Significant progress has been made in modelling the propagation effects and work is now in hand to incorporate the results into tools to aid the planning and operation of HF radio systems operating at northerly latitudes.

\subsection{INTRODUCTION}

Large deviations in the direction of arrival of ionospherically propagating radio signals from the Great Circle Path (GCP) have serious implications for the planning and operation of communications and radiolocation systems operating within the HF-band. Perhaps the most obvious example lies in the operation of radiolocation systems which usually operate by measuring the direction of arrival at several receiving sites. The location of the transmitter is then estimated from the intersection of the individual lines of bearing from each receiving site, and deviations from the GCP will therefore adversely affect the estimate of the transmitter location. The importance of off-great circle propagation extends beyond radiolocation to almost any HF system which employs directional antennas. With these systems, there is a significant possibility that performance will be degraded at times when the supported propagation path is in directions well displaced from the main lobe of one or both of the transmitting or receiving antennas.

Deviations of a few degrees from the great circle direction are associated with tilts due, for example, to the solar terminator and to travelling ionospheric disturbances (Jones and Reynolds, 1975). Very large deviations are particularly prevalent in the northerly regions where signals often arrive at the receiver with bearings displaced from the great circle direction by up to $\pm 100^{\circ}$ or more. Within the mid-latitude trough, these large deviations from the GCP arise from the electron density depletion and the consequent reduction in MUF along the great circle path. Propagation may then occur by scatter from irregularities in the north wall of the trough or within the auroral zone (which may be coincident) or by reflection from one or both of the tilted trough walls (Rogers et al., 1997; Stocker et al., 2002) or via ground/sea scatter (Stocker et al., 2003b). Within the polar cap, off great circle 
propagation is attributed to the presence of convecting patches and arcs of enhanced electron density (Warrington et al., 1997). It is important to note that the off-great circle mechanisms give rise to propagation at times which is not predicted by current prediction codes, and that techniques to account for this type of propagation are therefore required.

The situation is further complicated since, in addition to the large scale tilts which cause gross deviations of the signal from the great circle direction, irregularities in the electron density distribution cause signals associated with each propagation mode to arrive at the receiver over a range of angles in both azimuth and elevation. Such directional spread of the received signal energy is an important parameter to be considered in the design of multi-element receiving arrays and the associated signal processing methods used, for example, in radiolocation or adaptive reception systems. It is often assumed in the design of such systems that the signal environment comprises a limited number of specularly reflected signals arriving at the antenna array from well defined directions. However, for northerly paths, this is often not the case, and azimuthal standard deviations of several tens of degrees have been measured over polar cap paths (Warrington, 1998).

A common feature of northerly HF propagation is the large Doppler and delay spreads imposed on the signal. The magnitude of these effects is such as to severely limit the data throughput achievable in HF communications systems due to current technological limitations in modem design (Angling et al., 1998). The large Doppler spreads are often associated with directional spreading and recent work (Warrington et al., 2000) has indicated that adaptive beam/null steering from an array of antennas can be employed to exploit the directional spreading effects to reduce the apparent Doppler spread at the modem input.

Various measurements of off great-circle propagation effects over a range of northerly paths and their interpretation have been undertaken over a number of years by the authors and their colleagues, some of which is referred to above. Significant progress has been made over the COST 271 period and the aim of this paper is to summarise the important results of this research, to consider work in progress aimed at further improving our understanding of the high latitude propagation mechanisms, and to report on methods being developed for taking these propagation effects into account when designing and operating HF radio systems. By necessity, the discussion of individual aspects in this paper is brief and the reader is referred to several papers which consider individual topics in more detail.

\subsection{Propagation along the trough}

A $1400 \mathrm{~km}$ link along the mid-latitude trough (see fig. 14.1) was established with a transmitter located in Uppsala, Sweden and the receiver in Leicester, U.K. The transmitter operated on a 3 min cycle during which time transmissions were made in sequence on six frequencies in the range 4 to 18 MHz. A multi-channel receiver system connected to a spaced aperture antenna array capable of measuring the signal characteristics as a function of Time Of Flight (TOF), Doppler frequency, Doppler spread and also, by application of a superresolution DF algorithm to the multichannel data, direction of arrival. In addition, a BR communications chirpsounder was deployed at the sites to provide oblique ionograms to aid interpretation of the measurements. The systems were operated at all times of day over the course of just over a year, and results mainly obtained during 2001 are presented here. Further details of the experiment, measurements and results are given by Siddle et al. (2004a,b).

\subsubsection{Example measurements}

Presented in fig. 14.2 are the TOF and azimuthal direction of arrival measurements made over three days in November 2001 at $10.4 \mathrm{MHz}$. The daytime signals frequently exhibit 1-hop, 2-hop and 3-hop $F$-region propagation along the GCP, with a low Doppler spread and a relatively high signal 


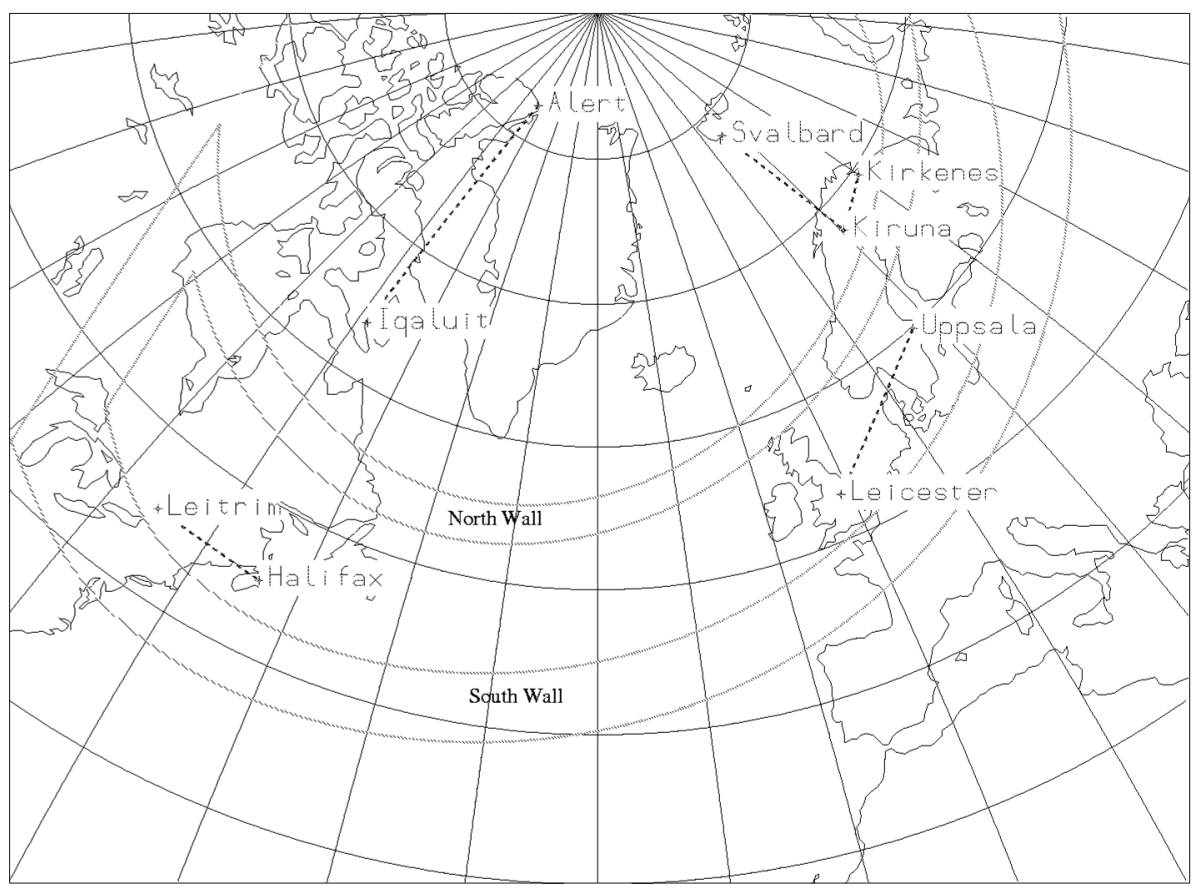

Fig. 14.1. Map showing the propagation paths discussed in this paper. Also shown is the position of the trough as estimated by the model of Halcrow and Nisbet (1977) for 00:00 UT on 11th March 2001, $K p=3$. The four lines indicate the outer and inner edges of the north and south walls.
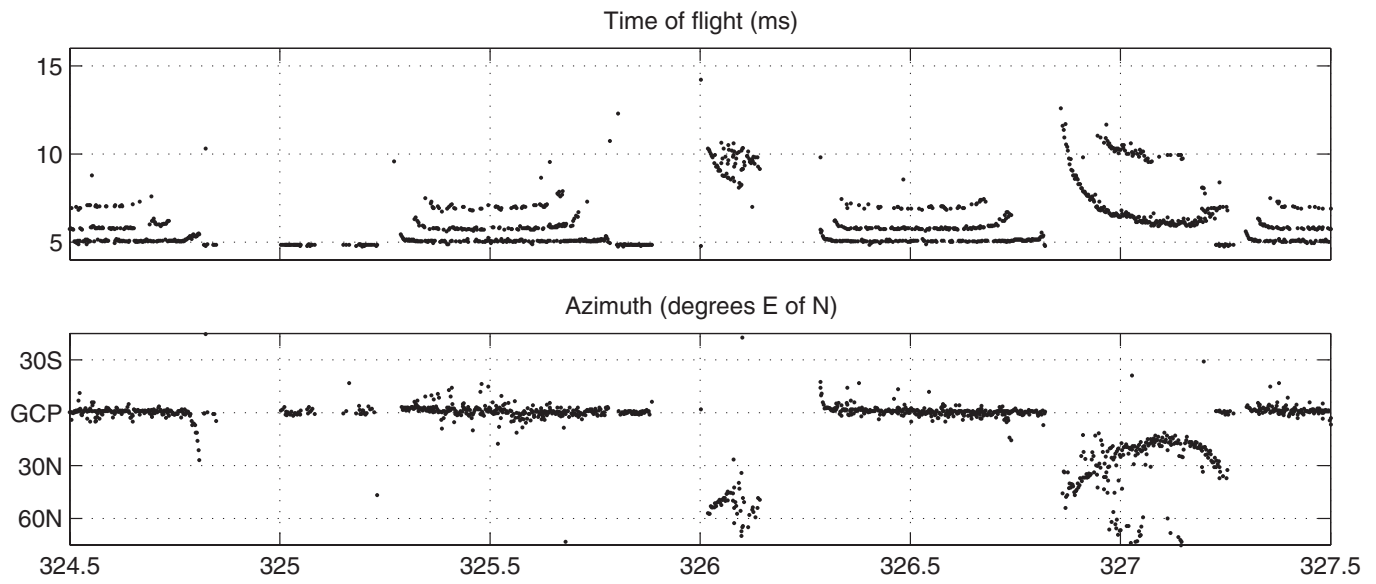

Fig. 14.2. Measurements made at $10.4 \mathrm{MHz}$ between noon on 20th November 2001 (day 324) and noon on 23rd November 2001 (day 327). The upper panel represents the time of flight (ms), and the lower panel the azimuth of the strongest mode $\left({ }^{\circ}\right)$. 
strength reduced only by $D$-region absorption in the middle of the day. At night, a wider variety of effects are apparent. On the first night (day 324-325), the daytime trace deviates northwards at sunset and is then replaced by intermittent $E$-region propagation along the GCP. The signal strength is about $20 \mathrm{~dB}$ lower than the daytime value and Doppler spread is low. On the second night (day 325-326), just after midnight, propagation occurs via a mode with a relatively long TOF (8-10 ms) and direction of arrival $40^{\circ}-60^{\circ}$ to the north. The signal is around $20 \mathrm{~dB}$ weaker than the daytime values with a Doppler shift of up to about $5 \mathrm{~Hz}$. The most striking behaviour can be observed on the third night (day 326-327) where a propagation mode appears at a long TOF (up to $13 \mathrm{~ms}$ ) at about 20:30 UT. Over the next $6 \mathrm{~h}$, the TOF decreases reaching a steady value of about $7 \mathrm{~ms}$ by 02:30 UT. At times around midnight a weaker, second mode at a longer delay $(\sim 11 \mathrm{~ms})$ is present. As the TOF decreases, the bearing deviation from the GCP decreases from about $40^{\circ}$ north at 20:30 UT to $20^{\circ}$ north at 02:30 $\mathrm{UT}$, the elevation angle increases from around $10^{\circ}$ to $15-20^{\circ}$ and the signal strength attains a level about $10 \mathrm{~dB}$ below the daytime, on-GCP value. The rapid decrease in TOF is accompanied by a strong positive Doppler shift $(\sim 20 \mathrm{~Hz})$. The Doppler spread is higher than daytime values until the direction of arrival deviates northwards (around 03:00 UT) when it is about the same as during the day.

\subsubsection{Occurrence statistics}

The percentage of nights on which various features are seen for the four central frequencies in the experiment is given in table 14.I. The seasons are defined according to ITU Recommendation P1148-1 (ITU, 1997), with spring including all of March and April, summer including May to Au-

Table 14.I. Occurrence statistics by mode and bearing for 7.0, 10.4, 11.1 and $14.4 \mathrm{MHz}$ signals during 2001 (all figures represent percentage of nights).

\begin{tabular}{|c|c|c|c|c|c|}
\hline & \multicolumn{2}{|c|}{ Observations } & \multicolumn{3}{|c|}{ Direction of arrival } \\
\hline & No propagation & Long TOF & GCP & North & South \\
\hline \multicolumn{6}{|l|}{$7.0 \mathrm{MHz}$} \\
\hline Spring & 8 & 31 & 82 & 41 & 2 \\
\hline Summer & 0 & 4 & 99 & 11 & 4 \\
\hline Autumn & 0 & 17 & 95 & 38 & 4 \\
\hline Winter & 8 & 64 & 47 & 80 & 1 \\
\hline \multicolumn{6}{|l|}{$10.4 \mathrm{MHz}$} \\
\hline Spring & 35 & 42 & 41 & 48 & 0 \\
\hline Summer & 2 & 21 & 91 & 37 & 2 \\
\hline Autumn & 13 & 60 & 55 & 69 & 0 \\
\hline Winter & 36 & 57 & 3 & 57 & 0 \\
\hline \multicolumn{6}{|l|}{$11.1 \mathrm{MHz}$} \\
\hline Spring & 61 & 35 & 18 & 31 & 0 \\
\hline Summer & 3 & 29 & 93 & 39 & 2 \\
\hline Autumn & 34 & 58 & 23 & 57 & 0 \\
\hline Winter & 44 & 55 & 1 & 47 & 0 \\
\hline \multicolumn{6}{|l|}{$14.4 \mathrm{MHz}$} \\
\hline Spring & 96 & 4 & 0 & 4 & 0 \\
\hline Summer & 65 & 7 & 37 & 8 & 0 \\
\hline Autumn & 82 & 16 & 0 & 13 & 0 \\
\hline Winter & 76 & 18 & 0 & 14 & 0 \\
\hline
\end{tabular}


gust, autumn September and October, and winter November to February. In deriving these statistics, propagation is deemed to have occurred if any readily identifiable trace, other than sporadic $E$, is seen between 21:00 and 03:00 UT. However, during some intervals, the data quality was not sufficient to categorise the direction of arrival and these together with the short-lived deviations that sometimes occur briefly at dawn and dusk (see e.g., sunrise on day 326 in fig. 14.2) have been excluded. The nighttime feature observed on day 326-327, a long TOF accompanied by northerly bearing deviations of up to $50^{\circ}$ and Doppler shifts is a relatively common occurrence in the non-summer months. During the summer, the deviation in azimuth tended to be much smaller $\left(<5^{\circ}\right)$ but still generally to the north. Deviations to the south were very rare, small in angle and short-lived.

\subsubsection{Comparison with VOACAP predictions}

In order to compare the experimental results with the predictions produced by VOACAP, occurrence statistics have been produced for the TOF, azimuth and elevation measurements by hour and month. The results for summer (June) and winter (December) 2001 are presented in fig. 14.3. The VOACAP predictions were obtained using method 25 in order to obtain information on each of the
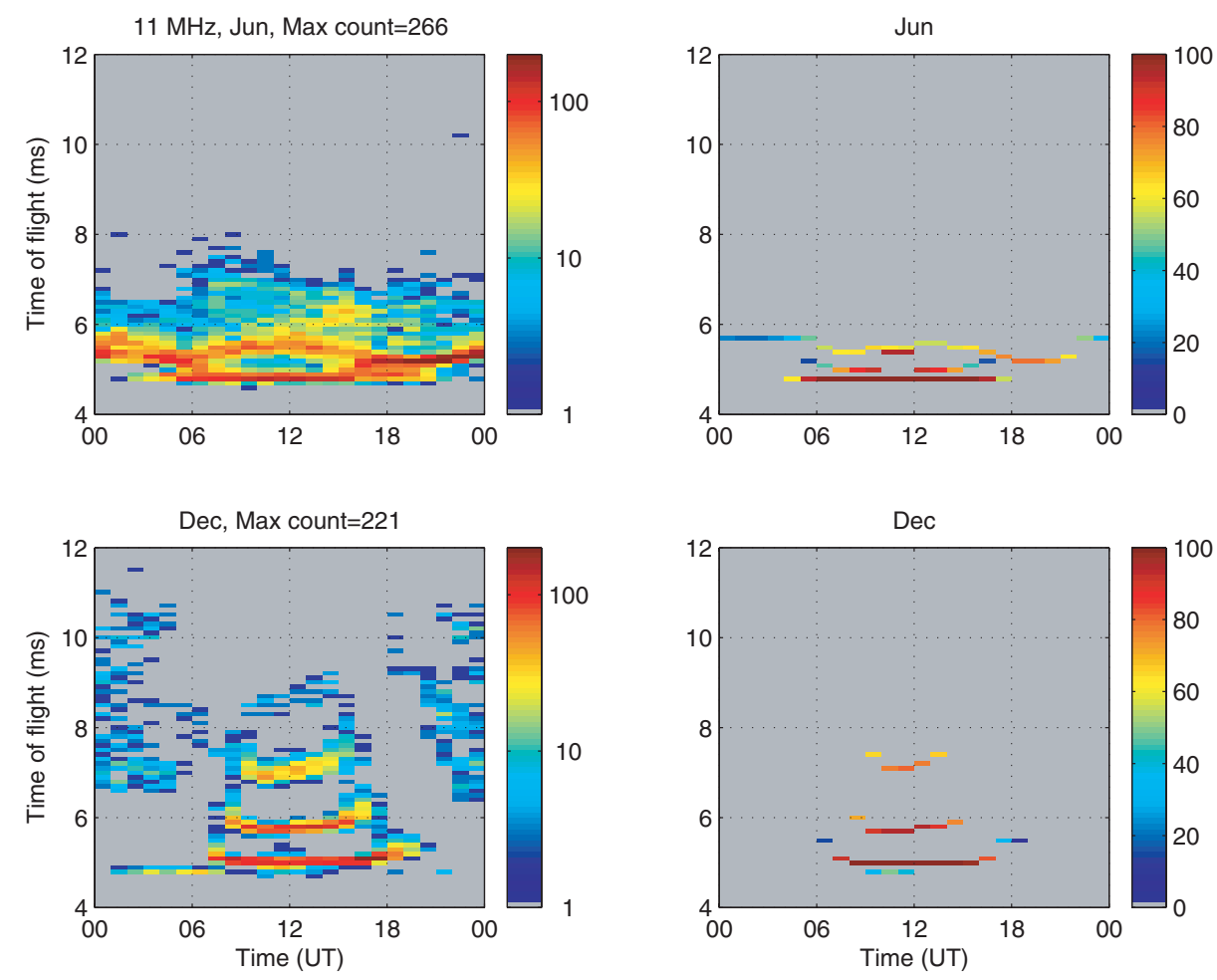

Fig. 14.3. Time of flight versus time of day for a signal of $11.1 \mathrm{MHz}$ in summer (top panels) and winter (bottom panels). The left-hand panels are derived from experimental data. The colour scale indicates the number of observations (i.e. a measure of the probability of occurrence). The right-hand panels are derived from VOACAP predictions. The colour scale gives the percentage of days for which the mode MUF is above the operating frequency. 
potential propagation modes. Only modes for which the CNR exceeds $0 \mathrm{~dB}$ and the probability of occurrence is non-zero are shown. The $0 \mathrm{~dB}$ CNR threshold was selected since this generally leads to a good agreement between the observations and predictions for daytime conditions.

The hourly values of the observed and predicted TOF for June and December 2001 for a frequency of 11.1 MHz are presented in fig. 14.3. In June, VOACAP predicts three modes in the daytime ( $1 E$, $1 F 1$, and $1 F 2$ ) and a single mode at night at a slightly longer delay. The observations are generally in good agreement with the predictions, although the resolution of the measurements $(0.1 \mathrm{~ms})$ precludes separating the $1 E$ - and $1 F 1$-modes in the daytime, and the delay for the night-time mode is slightly longer than that predicted. For the winter month, the daytime propagation is well predicted by VOACAP with the $1 F 2$-, 2F2-, and 3F2-modes clearly visible. At night, VOACAP does not do so well, with the propagation at long TOF (7 to $11 \mathrm{~ms}$ ) that is present in the observations from about 20:00 UT through to 06:00 UT not being reproduced. Since the sporadic $E$ model that is available in VOACAP was not utilised, it was not expected that the sporadic $E$-layer observed at a TOF of just below $5 \mathrm{~ms}$ from about 01:00 UT to 08:00 UT would be reproduced in the prediction results.

Additional information on the comparison between the VOACAP predictions and the measurements is given by Stocker et al. (2003a, 2004).

\subsubsection{Delay and Doppler spread parameters}

As may be expected, the measured spread characteristics are strongly dependent upon time of day and season since the trough is a night time feature which occurs predominantly during the winter. Both the multipath and Doppler spreads are markedly less in summer than in winter, during which time a strong diurnal variation is apparent (see fig. 14.4). Tabulated values of the cumulative distributions of the multipath and Doppler spreads measured on each of the six frequencies employed in the experiments for day and night time in winter are presented in table 14.II.

For the winter period, it is interesting to consider the spread parameters separately for signals arriving close (taken as between $41^{\circ}$ and $48^{\circ}$ measured clockwise from north) to the great circle direction and for signals arriving well to the north (taken as between $0^{\circ}$ and $35^{\circ}$ ) of the great circle direction. It is evident when catagorising the data in this manner that the large Doppler spreads observed at night are associated with propagation to the north of the GCP, i.e. when the signal is reflected from the disturbed ionosphere forming the north wall of the trough or within the auroral oval.

For further details see Warrington and Stocker (2003).

\subsubsection{Theoretical considerations}

For the purposes of this study, an empirical model of the trough given by Halcrow and Nisbet (1977) is employed. This model is based on satellite measurements, and is parameterised by $K p$, may be used to predict the latitude of the top and bottom of the north and south walls of the trough as a function of local time. $K p$, local time and the solar zenith angle are used to give the location of the sunrise and sunset walls of the trough.

Shown as bold lines in fig. 14.5 are the latitudes of the top (i.e. unperturbed) and bottom (i.e. fully perturbed) edges of the north and south trough walls, as derived from the Halcrow and Nisbet model. Also indicated in this figure are estimates of the latitude of the reflection points inferred from the observations. These estimates were obtained from the measurements of TOF, azimuth and elevation for signals identified as single-hop reflections assuming a single specular reflection, linear propagation and taking into account the curvature of the earth. Agreement between observations and model is evident in this figure.

A ray tracing study has also been undertaken (Siddle et al., 2004b), the main parameters and results of which are briefly summarised below. 

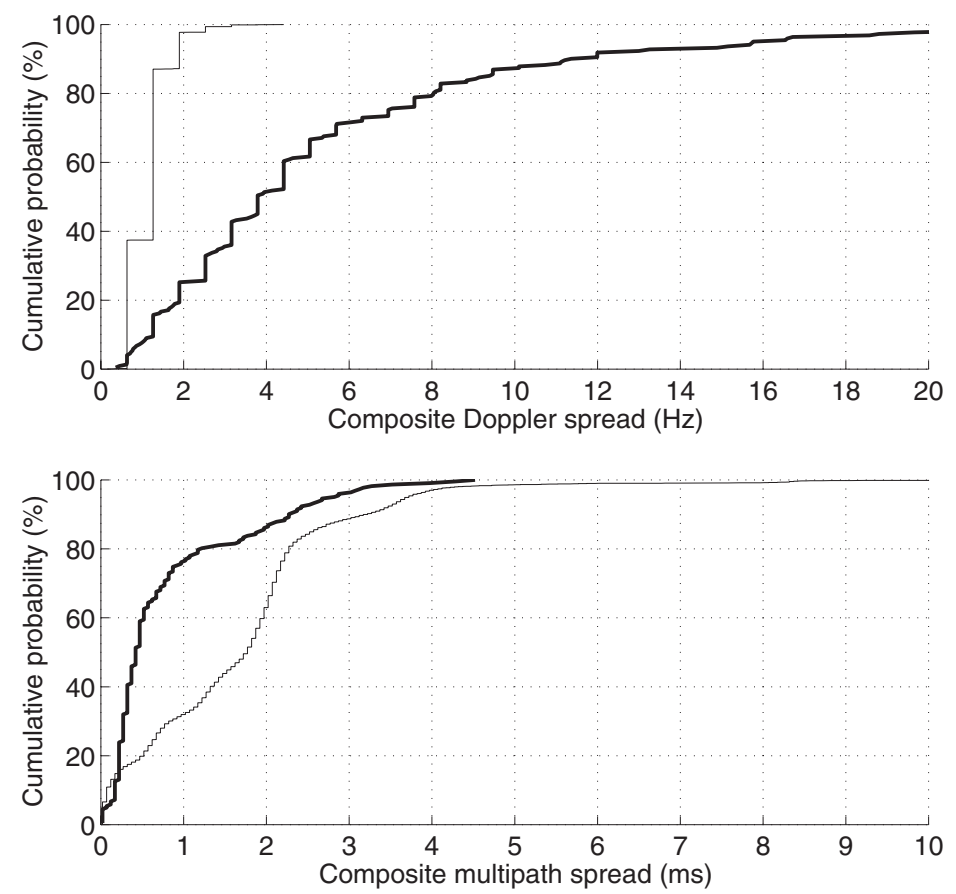

Fig. 14.4. Cumulative probability plots of composite Doppler spread (upper frame) and composite multipath spread (lower frame) measured at $11.12 \mathrm{MHz}$ during winter. The bold curves are night time and the thin curves for day time.

Table 14.II. Median and 95\% occurrence values of spreads during winter (November, December and January) day (09:00-15:00 UT) and night (21:00-03:00 UT).

\begin{tabular}{lccccccc}
\hline \hline Frequency (MHz) & & 4.6 & 7.0 & 10.4 & 11.1 & 14.4 & 18.4 \\
\hline Day & & & & & & & \\
Doppler & $50 \%$ & - & 1.3 & 1.3 & 1.3 & 1.3 & 0.6 \\
\multirow{2}{*}{ Multipath } & $95 \%$ & - & 1.3 & 1.9 & 1.9 & 1.9 & 1.9 \\
& $50 \%$ & - & 0.9 & 1.5 & 1.8 & 0.7 & 0.0 \\
Effective multipath & $95 \%$ & - & 3.1 & 3.4 & 3.7 & 2.1 & 1.5 \\
& $50 \%$ & - & 0.2 & 0.3 & 0.3 & 0.1 & 0.0 \\
Night & $95 \%$ & - & 1.1 & 1.4 & 1.5 & 0.9 & 0.6 \\
Doppler & & & & & & & \\
& & & & & & & - \\
Multipath & $50 \%$ & 0.6 & 1.3 & 3.3 & 3.8 & - & - \\
\multirow{2}{*}{ Effective multipath } & $95 \%$ & 1.9 & 1.9 & 12.8 & 15.7 & - & - \\
& $50 \%$ & 0.1 & 0.1 & 0.3 & 0.4 & - & - \\
\hline
\end{tabular}



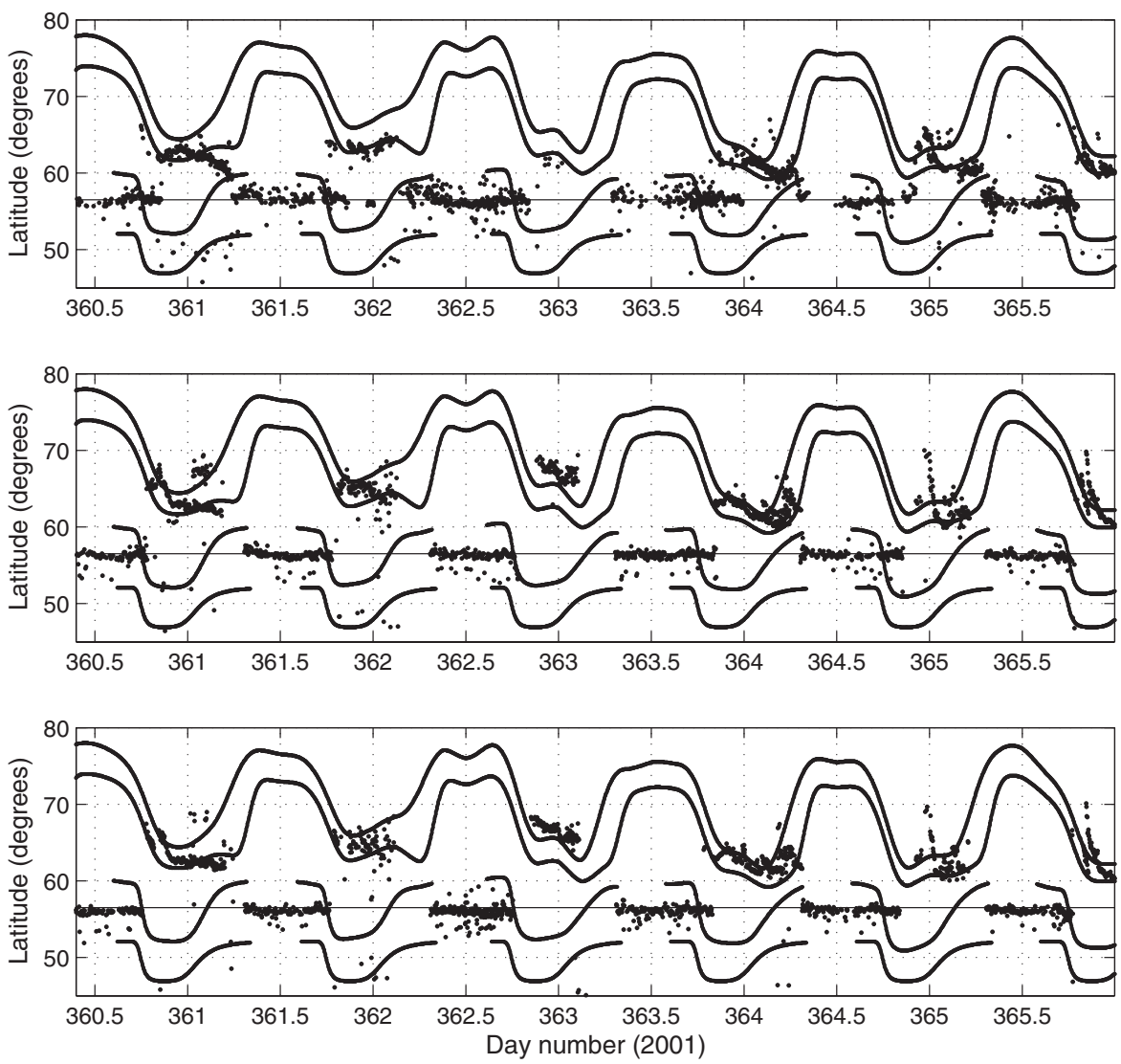

Fig. 14.5. The latitude of the calculated point of virtual reflection and of the model trough walls between noon on 26th December 2001 (day 360) and the end of 31st December 2001 (day 365). From top to bottom: frequency 7.0, 10.4 and $11.1 \mathrm{MHz}$. The horizontal line at $56.5^{\circ} \mathrm{N}$ represents the latitude of the mid-point of the GCP.

The Halcrow and Nisbet model was used as a basis for the position of the trough walls. Initial studies using an unmodified trough model were unable to reproduce the observations. Since the smooth walls produced by the model are unrealistic, smaller-scale structures were added to the modelled electron density profile which produced a landscape of patches along each wall, which were elongated in the direction longitudinal to the trough. This created small areas of higher density gradient than exist in the original model, and which enhance the wall's ability to reflect rays. The maximum depletion of the trough was set according to the phase of the solar cycle. Typically, a reduction in electron density by $20-30 \%$ was used for times of maximum sunspot number, and a reduction of $60 \%$ for low sunspot number. These values represent averages derived from ionograms from sounding stations under the trough and close to the southern edge, and they differ markedly from the constant reduction of $75 \%$ assumed in the Halcrow and Nisbet model. It should, however, be noted that the Halcrow and Nisbet model is based on top-side soundings, whereas we are considering the bottom-side ionosphere.

A model of the auroral oval was also included in the simulations. In the model the equatorward edge of the auroral oval was coincident with the poleward edge of the trough, although it should be 
noted that the oval is sometimes to the north of the trough wall. Although precipitation is known to vary over the solar cycle, this variation was not included.

For the Uppsala to Leicester path (see fig. 14.1), the tilts associated with the walls of the trough had little effect. The deviations in direction of arrival could only be accounted for by scatter from irregularities in the auroral zone. This is in marked contrast to the simulations of measurements made in Canada over a path from Halifax, Nova Scotia to Leitrim, Ontario close to sunspot minimum in which reflections from both north and south walls of the trough occurred. This latter observation is attributed to the enhanced electron density depletion within trough at this phase of the solar cycle.

\subsection{OfF-great CIRCle PROPAGation Within the POlar CAP}

Measurements were made over the $2100 \mathrm{~km}$ polar cap path from Iqaluit to Alert (see fig. 14.1) for a period of approximately 2.5 years between December 1993 and May 1996 (see Warrington et
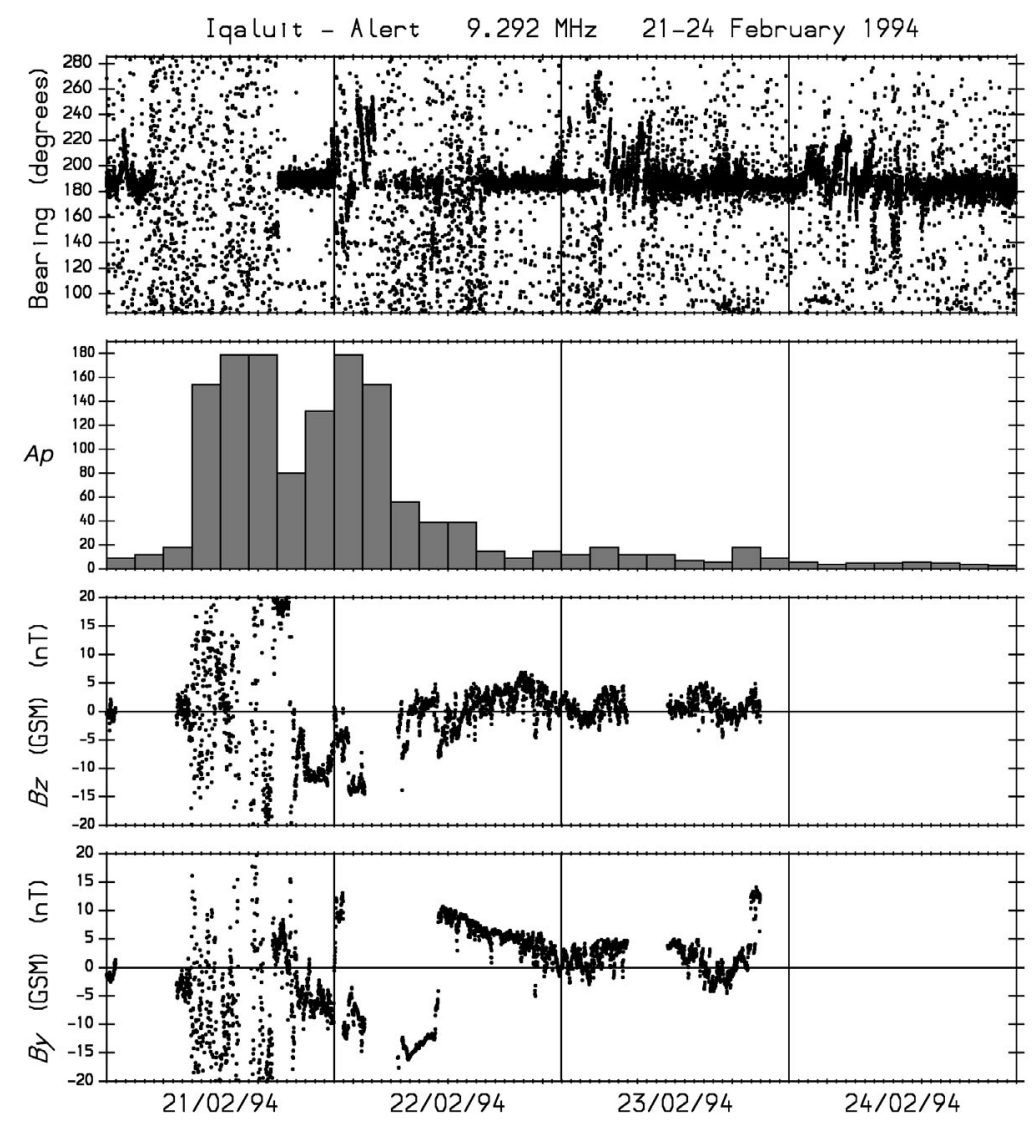

Fig. 14.6. Bearings measurements for the $9.292 \mathrm{MHz}$ transmission from Iqaluit received at Alert for the period 21st-24th February 1994. Three-hourly $A p$ values and the IMF $B y$ and $B z$ values are also shown. Bearings are measured in degrees clockwise from north, and the times/dates are in UT. 
al., 1997). During the winter and equinoctial months, there is an underlying tendency for propagation to deviate to the west of the great circle path (high bearing angles) in the evening sector (local midnight at the GCP mid-point is 04:30 UT) with propagation returning from the east of the GCP (low bearing angles) in the morning. This probably arises from very large scale ionospheric gradients in the polar cap associated with the solar terminator. Of particular note is the occurrence of rapid bearing swings with periods of about $30 \mathrm{~min}$ which are attributed to the presence of convecting patches and arcs of enhanced electron density.

An example period illustrating the rapid bearing swings observed at $9.292 \mathrm{MHz}$ for the period 21st-24th February 1994 is presented in fig. 14.6 together with values of the 3-hourly Ap index and the $B y$ and $B z$ IMF parameters. A geomagnetic storm is evident on 21st-22nd February. The principal bearing swings on the night of the 21st-22nd February, a period of southward IMF and high $A p$ values, have a decreasing bearing angle and occur in the six hour period before local midnight (04:30 UT), whereas the principal bearing swings on the following night, a period of northward IMF and low $A p$, have an increasing bearing and occur principally in the hours following local midnight.

Reference to the expected convection flow patterns (see Lockwood, 1993) suggests that when the IMF is directed southward $(B z<0)$, patches of ionisation drifting anti-sunwards would lead to a preponderance of decreasing bearing angle swings in the pre-midnight hours and increasing bearing angle swings in the hours after midnight. Figure 14.6 shows this to be the case where $B y<0$, although where $B y>0$ there is a distinct lack of decreasing swings in the pre-midnight sector. It is important to note, however, that the convection flows given by Lockwood are sketches of the overall form of what may be expected. On any particular occasion, the actual flow patterns may differ significantly from those in the illustration. When the IMF is directed northwards $(B z>0)$, the principal large scale electron density structures within the polar cap ionosphere are sun-aligned arcs (plasma striations extending for thousands of kilometres in the trans-polar noon-midnight direction, but much narrower with scales around $100 \mathrm{~km}$ in the dawn-dusk direction) (Buchau et al., 1983). A series of arcs drifting steadily across the polar cap from dawn to dusk would lead to the expectation that increasing bearing swings would be observed during the time sector 18:00 to 06:00 LT, with the largest swings expected in the midnight sector. Decreasing bearing swings would be observed in the local time sector 06:00 to 18:00 with the largest swings in the noon sector.

\subsubsection{Simulation}

Considerable progress has been made with the development of a ray-tracing model which accurately reproduces many of the features observed in the experimental measurements to a level well beyond that which we had originally anticipated (see Zaalov et al., 2003). A major outcome of the raytracing simulations is that paths other than those subject to experimental investigation can readily be assessed. Consideration is now being given to incorporating the results of the ray tracing studies into a rule-base to allow the effects to be quickly evaluated (the ray-tracing is computationally intensive and is not suited to incorporation in real-time prediction codes).

The simulations make use of a numerical ray tracing code (Jones and Stephenson, 1975) to estimate the ray paths through a model ionosphere comprising two Chapman layers, the main parameters of which (critical frequency, critical height, vertical scale height of each layer) are based on values obtained from the International Reference Ionosphere (IRI) (Bilitza, 1990). The IRI model was chosen since it provides a reasonable model of the background ionosphere from the point of view of long term predictions but it is, of course, possible to use other models or even experimental data when available. Marked day to day deviations from the average background ionosphere are to be expected. In order to introduce some flexibility in the ionospheric models and to significantly improve the computational speed, some analytical approximations were made to the longitudinal and latitudinal elec- 
tron density gradients based on the magnitudes of the critical frequencies of the main layers $(E, F 2)$ at five reference points (the North Pole, two points near midnight and two points near noon). Localised, time varying perturbations in the electron density are then applied to the background model to represent the convecting patches and arcs of enhanced electron density.

\subsubsection{Convecting patches}

Patches of enhanced electron density associated with high geomagnetic activity are modelled as an arbitrary number of Gaussian distributions with approximately equal longitudinal and latitudinal scale. The temporal evolution of the patches relative to the propagation path is simulated by means of a convection flow scheme coupled with the rotation of the Earth beneath the convection pattern, the precise form of which depends upon the components of the IMF. In practice, the shape, size and number of patches in the convection flow area depends upon many geophysical parameters, not only upon the instantaneous values but also upon their history. By using up to four vortices based on the modelled convection flow patterns associated with the various IMF orientations presented by Lockwood (1993), many realistic situations may be simulated.

Examples of the azimuth deviations produced by the presence of patches are given in fig. 14.7 for convection patterns associated with values of $B y>0$. In this example, there were 28 patches of enhanced electron density with a maximum critical frequency of approximately $9 \mathrm{MHz}$. Only those patches relatively close to the propagation path influence the structure of the received signal, but as the patches evolve and move following the modelled convection flow patterns, the number and positions of those patches influencing the signal changes. An ionogram simulated for an ionosphere where enhanced patches are present are given in fig. 14.8. This is an example of a 'nose extension' (i.e. the fea-

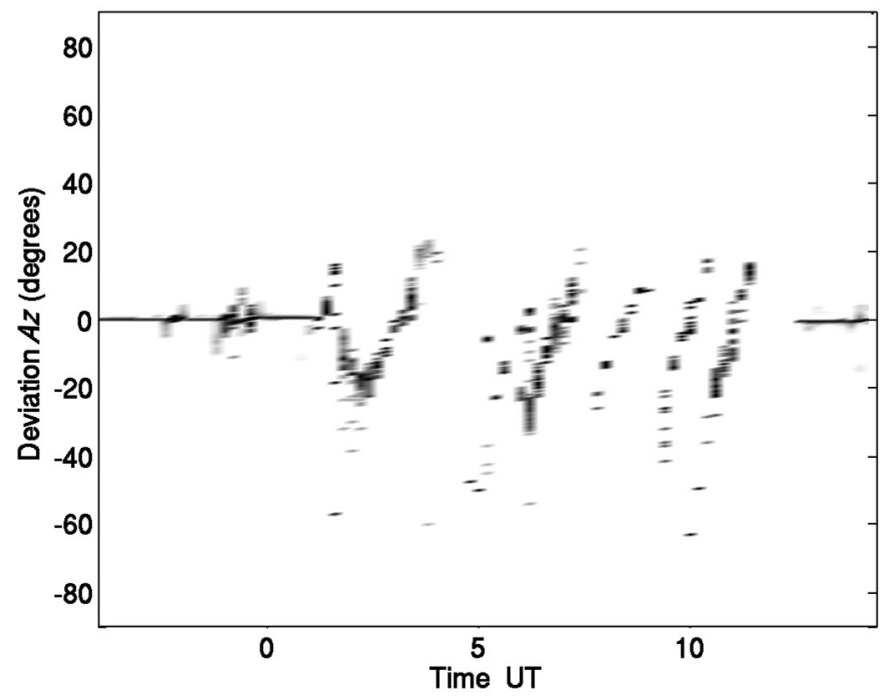

Fig. 14.7. Time history of the direction of arrival (azimuth only) of a $9.3 \mathrm{MHz}$ signal propagating through a model ionosphere containing patches of enhanced electron density for $B y>0$. 

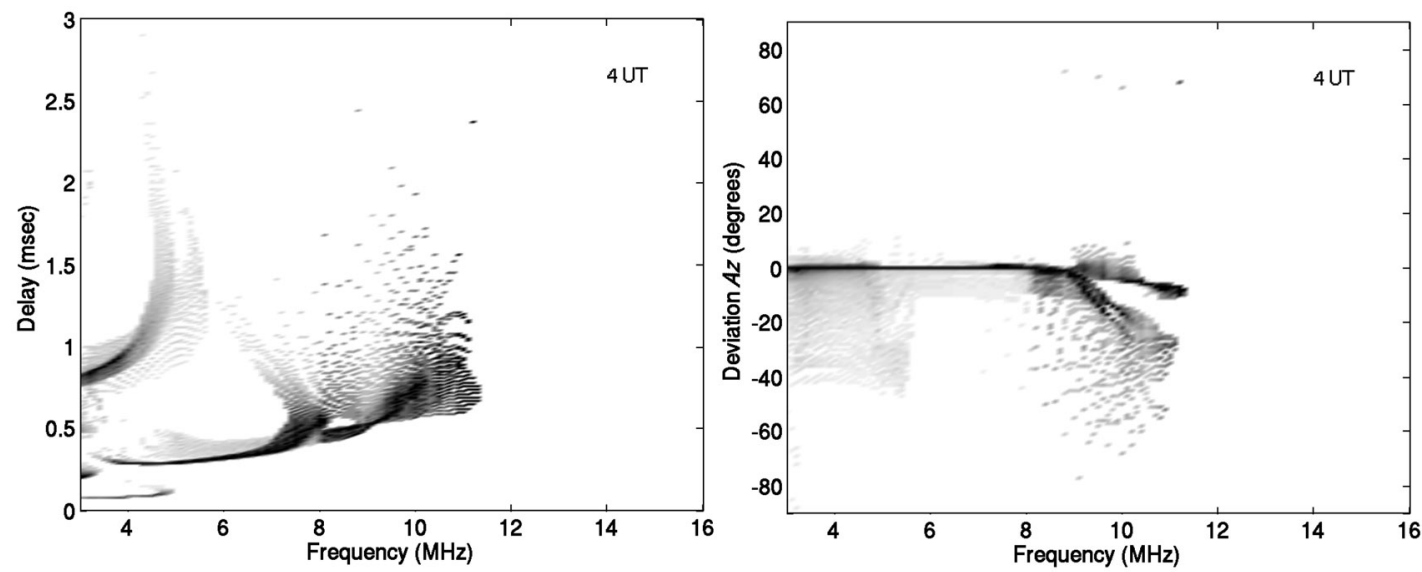

Fig. 14.8. Oblique ionogram produced by ray tracing simulations through an ionosphere containing patches of enhanced electron density.

ture above about $8 \mathrm{MHz}$ ) to the normal ionogram trace in which energy is received at frequencies above the junction frequency of the high and low angle magneto-ionic components. These features are frequently seen in the experimental observations. The lower panel of fig. 14.8 indicates the azimuthal direction of arrival of the signal energy as a function of frequency, and it is interesting to note the significant deviation from the great circle path at frequencies above the junction frequency. In other simulated ionograms, the nose-extension feature becomes detached from the main trace, and again this is a feature commonly present in the ionograms obtained in practice (see Rogers et al., 2003).

\subsubsection{Sun aligned arcs}

The shape of each sun-aligned arc is defined within the model by a small number of three dimensional Gaussian perturbations in electron density of different spatial scales (altitude, longitude and latitude) randomly distributed near to the centre of the arc. Several Gaussian perturbations were combined in defining the shape of each modelled arc in order to prevent the shapes of the arcs being too stylised. For all arcs away from close proximity to the dawn or dusk auroral oval, the plasma strands are elongated for several hundreds or thousands of kilometres with a latitudinal scale which is significantly larger than the longitudinal scale. Many such arcs can be included in the simulation with their positions being randomly distributed in an area centred on the geomagnetic pole and bounded by the auroral oval. The magnitude of the electron density perturbation of each of the elements forming the arcs is randomly distributed about a specified average value. Evolution of the structures relative to the propagation path is determined by the rotation of the Earth beneath the arcs and by the movement of the arcs in the dawn-dusk direction.

\subsection{Propagation over a trans-oval path}

An experimental campaign is currently being conducted with a receiver system capable of measuring the delay and Doppler spread characteristics and the directional structure of the received signals 
at Kiruna in Northern Sweden. Transmitters are located on Svalbard (a path length of $1152 \mathrm{~km}$ ) and near to Kirkenes in northern Norway $(430 \mathrm{~km}$ ) (see fig. 14.1). Measurements have also been made for a short period over the path from Uppsala in southern Sweden $(880 \mathrm{~km})$.

The radiated signals comprise sequences of 13-bit Barker coded PSK pulses. Since the transmitter and receiver systems are synchronised to GPS, the time of flight of the signals may be determined. The signals are received on a six-element spaced aperture antenna array, the individual elements of which are connected to individual inputs of a multi-channel receiver. The signal samples received on each antenna within the array are processed to provide a measure of the absolute times of flight of the propagating modes and their associated Doppler spectra. In this way, the signal may be split into complex amplitude components distinguished by time of flight, Doppler frequency and, since a multi-channel receiver with a spaced antenna array was employed, by antenna position. A superresolution direction finding algorithm may then applied to the spatially resolved information in order to estimate the directional structure of the signals.

One of the aims of this experiment is to relate the oblique propagation parameters to the measurements made with the CUTLASS and other SuperDARN radars. The identification of suitable well-defined relationships may enable the estimation of oblique signal characteristics for prolonged periods (i.e. those periods not covered by these experiments but covered by the other instrumentation) and over other paths by examination of the data produced by the continuously operating geophysical HF radars. It may therefore be possible to extend the results of this work to encompass the extensive geographical area covered by the SuperDARN radar network, of which the CUTLASS radars form an element.

A typical example of a series of measurements made at $11.2 \mathrm{MHz}$ on 30th September 2002 which exhibit Doppler spreading is presented in fig. 14.9. The upper frame of this figure shows the

$11 \mathrm{MHz}$, Svalbard-Kiruna
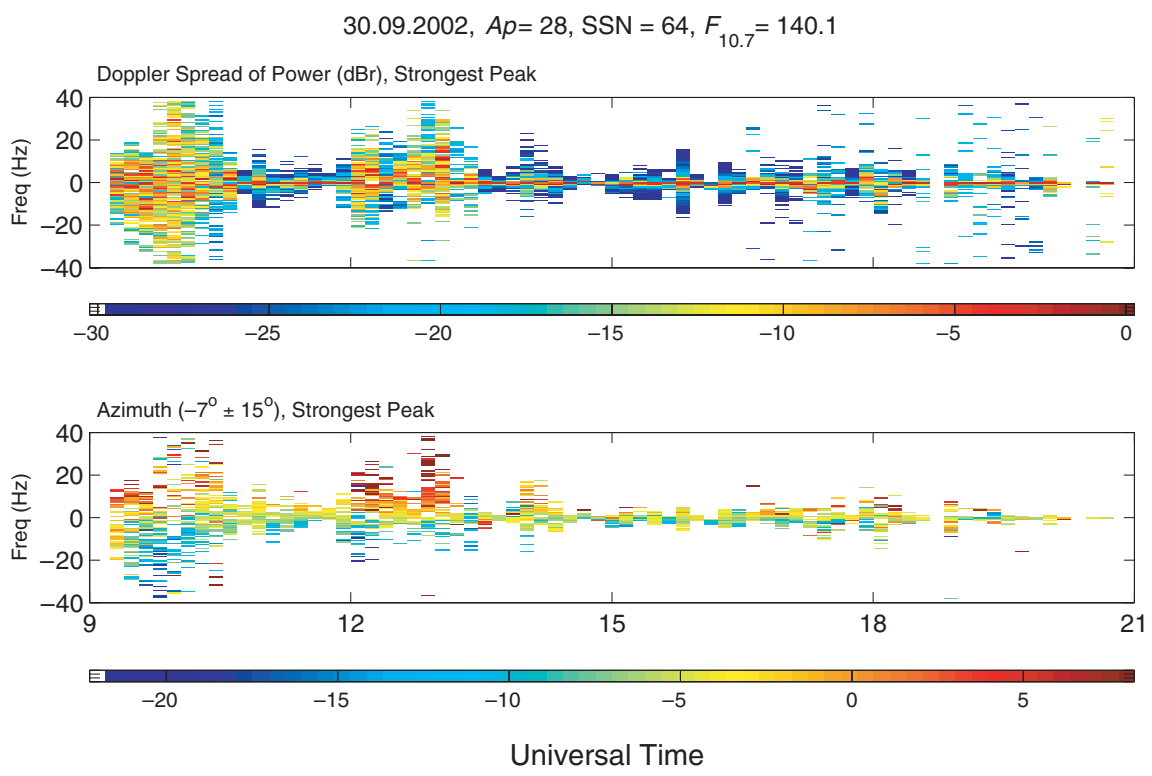

Fig. 14.9. Doppler spectrum (upper panel) and direction of arrival (lower frame) for the $11 \mathrm{MHz}$ signal received over the Svalbard to Kiruna path (30th September 2002). 


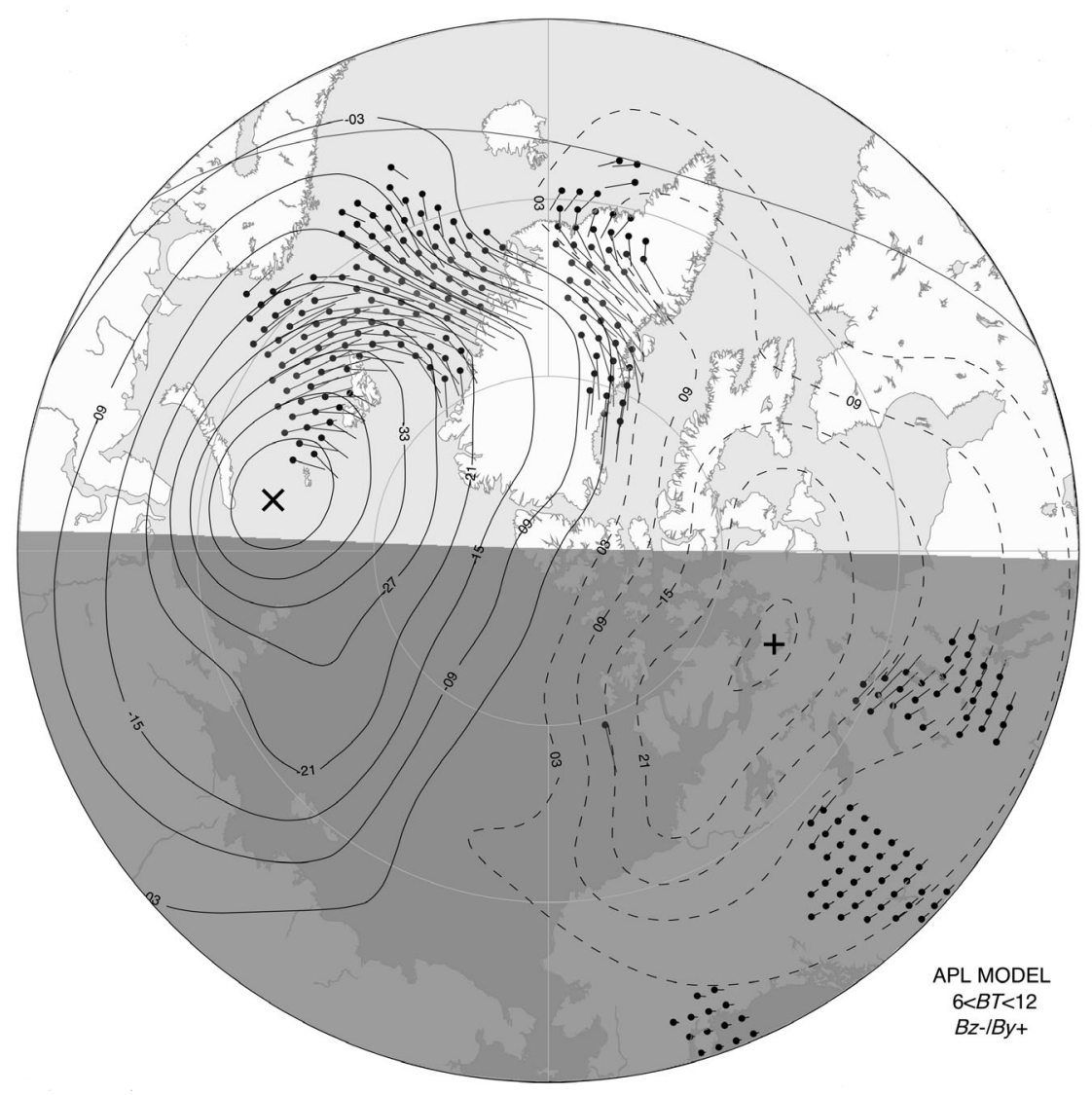

Fig. 14.10. Ionospheric convection flows estimated by the SuperDARN radars. 12:10 UT, 30th September 2003.

Doppler spectrum of the signal for the period 09:00 UT until 21:00 UT, whilst the lower frame shows the direction of arrival of each component in the Doppler spectrum. A marked relationship is evident between the Doppler frequency and the measured bearing. Signal components arriving at the receiver from directions to the east of the great circle path (high bearing angles) have positive Doppler shifts imposed, whereas signals arriving from the west of the great circle direction (low bearing angles) have negative Doppler shifts imposed. During this example period, the CUTLASS radars detected the presence of ionospheric irregularities moving with the convection flow at velocities of several hundred metres per second in an east to west direction at latitudes similar to the mid-point of the Svalbard to Kiruna path (see fig. 14.10). For irregularities drifting in a westerly direction, positive Doppler shifts would be imposed on any signal components scattered from irregularities to the east of the great circle direction where the motion of the scatterers was in a direction tending to shorten the path and negative Doppler shifts imposed on those scattered components to the west of the great circle path direction where the motion of the scatterers was in a direction tending to lengthen the path. The sense of the observed relationship between bearing and Doppler frequency is in good agreement with the radar observations of the flow direction. 


\subsection{DISCUSSION AND CONCLUDING REMARKS}

The results presented in this paper illustrate the importance of taking off-great circle propagation effects into account when planning and operating HF radio links. Often, these effects are considered as only being of importance in radiolocation (HF-DF) where deviations from the great circle path may result in significant (sometimes inter-continental) triangulation errors. It is also noteworthy that in systems employing directional antennas pointed along the great circle path that the signal quality may be degraded at times when propagation is via off-great circle propagation modes.

It is clear for the path geometries considered in this paper that when great circle propagation is not supported and the signals propagate via other mechanisms, the signal characteristics differ significantly from those associated with great circle propagation. In particular it is important to note the increased delay and Doppler spreads which are of relevance to the design and selection of HF radio modem protocols. Modems can be characterised (approximately) such as to have threshold values above which the error rate degrades to intolerable levels. For example, to achieve a bit error rate of $10^{-3}$ for a MIL-STD 110A modem operating at 1200 baud (note there may be differences between different implementations of the standard) the modem can tolerate Doppler spreads of up to around $7 \mathrm{~Hz}$ and multipath spreads of up to around $7 \mathrm{~ms}$. The levels of Doppler spreading observed over the path along the trough from Uppsala to Leicester during the winter often exceeds that which can be tolerated by this type of modem. Unsurprisingly, higher speed modems are less tolerant of channel dispersion than the lower speed waveforms.

Observations along the mid-latitude trough have been compared with predictions from VOACAP. The agreement between observation and predictions is generally very good during the day in both summer and winter, and at night during summer. The presence of off-great circle propagation during winter nights is not predicted by VOACAP when propagation is by scattering from irregularities in the trough wall or from the auroral oval which may be slightly to the north of the wall. For the Uppsala-Leicester path, propagation of this type occurs on over half of nights during the winter, but rarely during the summer. These off great circle modes represent an opportunity for communication at times at which it otherwise would not be possible, albeit at reduced SNR.

In order for these propagation effects to be properly taken into account in system design and operation, it is necessary for the propagation mechanisms to be fully understood and incorporated into prediction tools. The authors are currently working towards this, and significant progress has been made in modelling the off-great circle propagation effects (Zaalov et al., 2003). The results of the modelling are very reminiscent of the characteristics observed in the experimental measurements programmes, and enable the nature of off-great circle propagation effects to be estimated for paths which were not subject to experimental investigation. Although it is not possible to predict individual events, due to the unpredictable nature of the precise positions of polar patches and arcs, it is possible to predict the periods during which the large deviations are likely to occur, their magnitudes and directions. The modelling relies on ray tracing through model ionospheres which is computationally intensive. Consequently, it is not envisaged that any propagation prediction tools developed as part of this research will contain ray tracing elements. An alternative approach is being made whereby a large number of ray tracing results will be included in a data-base which will form part of a rule base for predicting the effects of off-great circle propagation on any path impinging on the northerly ionosphere.

\section{ACKNOWLEDGEMENTS}

The authors would like to thank the various organisations which have hosted the transmitting and receiving systems employed in this investigation - the Auroral Station in Adventalen, Svalbard, the Swedish Institute of Space Physics, Kiruna, the Swedish Meteorological Institute, Uppsala and the Norwegian Defence Research Establishment. The SuperDARN radars used in part of this study are 
supported by the Research Councils of the U.K., France, U.S.A., Canada and Japan, and we thank the PIs of the radars for the use of their data. The authors would also like to acknowledge support from the EPSRC and from the Canadian DND/DRDC.

\section{REFERENCES}

Angling, M.J., P.S. CAnnon, N.C. Davies, T.J. Willink, V. Jodalen and B. LundBorg (1998): Measurements of Doppler and multipath spread on oblique high latitude HF paths and their use in characterising data modem performance, Radio Sci., 33 (1), 97-107.

BILITZA, D. (Editor) (1990): International Reference Ionosphere 1990, Greenbelt, Maryland, U.S.A., NSSDC 90-22.

Buchau, J., B.W. Reinisch, E.J. Weber and J.G. Moore (1983): Structure and dynamics of the winter polar cap F-region, Radio Sci., 18, 995-1010.

HALCROW, B.W. and J.S. NisBeT (1977): A model of the F2 peak electron densities in the main trough region of the ionosphere, Radio Sci., 12, 815-820.

ITU (INTERNATIONAL TELECOMMUNiCATIONS UniON) (1997): Standardized procedure for comparing predicted and observed HF sky-wave signal intensities and the presentation of such comparisons, ITU-R Recommendation P1148-1.

JONES, T.B. and J.S.B. REYNOLDS (1975): Ionospheric perturbations and their effect on the accuracy of HF direction finders, Radio Electron. Eng., 45, 63-73.

Jones, R.M. and J.J. STEPHENSOn (1975): A Versatile Three Dimensional Ray Tracing Computer Program for Radio Waves in the Ionosphere, Office of Telecommunications, OT 75-76 (US Department of Commerce, Washington, U.S.A.).

Lockwood, M. (1993): Modelling the high latitude ionosphere for time varying plasma convection, Proc. Inst. Electr. Eng., Part H, 140 (2), 91-100.

Rogers, N.C., E.M. WARRINGTON and T.B. Jones (1997): Large HF bearing errors for propagation paths tangential to the auroral oval, IEE Proc. Microwaves Ant. Prop., 144 (2), 91-96.

Rogers, N.C., E.M. WARRINGTON and T.B. Jones (2003): Oblique ionogram features associated with off-great-circle HF propagation at high and sub-auroral latitudes, IEE Proc. Microwaves Ant. Prop., 150 (4), doi:10.1049/ip-map:20030552, 295-300.

Siddle D.R., A.J. Stocker and E.M. WARrington (2004a): The time-of-flight and direction of arrival of HF radio signals received over a path along the mid-latitude trough: observations, Radio Sci., 39, RS4008, doi: 10.1029/2004RS003049.

Siddle D.R., N.Y. ZaAlov, A.J. Stocker and E.M. WARrington (2004b): The time-of-flight and direction of arrival of HF radio signals received over a path along the mid-latitude trough: theoretical considerations, Radio Sci., 39, RS4009, doi: 10.1029/2004RS003052.

StOcKer, A.J., E.M. WARrington and N.Y. ZAAlov (2002): The effect of the mid-latitude trough on the direction of arrival and time-of-flight of HF radio signals, Acta Geod. Geophys. Hung., 37 $(2 / 3)$.

Stocker, A.J., D.R. SidDlE and E.M. WARRINGTON (2003a): Comparison between the measurement and prediction of HF radio signals propagating along the mid-latitude trough, in Proceedings of the IEE International Conference on HF Systems and Techniques, 145-149.

Stocker, A.J., E.M. WARrington and T.B. Jones (2003b): A comparison of observed and modelled deviations from the great circle direction for a $4490 \mathrm{~km}$ HF propagation path along the mid-latitude ionospheric trough, Radio Sci., 38 (3), 1045, doi:10.1029/2002RS002781.

Stocker, A.J., E.M. WARRINGTON and D.R. SidDle (2004): Comparison between the measured and predicted parameters of HF radio signals propagating along the mid-latitude trough and within the polar cap, Radio Sci. (submitted).

WARRINGTON, E.M. (1998): Observations of the directional characteristics of ionospherically propa- 
gated HF radio channel sounding signals over two high latitude paths, IEE Proc. Microwaves Ant. Prop., 145 (5), 379-385.

WARrington, E.M. and A.J. STOCKer (2003): Measurements of the Doppler and multipath spread of HF signals received over a path oriented along the mid-latitude trough, Radio Sci., 38 (5), 1080, doi:10.1029/2002RS002815.

WARRINGTON, E.M., N.C. Rogers and T.B. JONES (1997): Large HF bearing errors for propagation paths contained within the polar cap, IEE Proc. Microwaves Ant. Prop., 144 (4), 241-249.

WARrington, E.M., C.A. JACKSON and B. LUNDBORG (2000): Directional diversity of HF signals received over high latitude paths and the possibility of improved data throughput by means of spatial filtering, IEE Proc. Microwaves Ant. Prop., 147 (6), 487-494.

ZaAlov, N.Y., E.M. WARRINGTON and A.J. STOCKER (2003): The simulation of off-great circle HF propagation effects due to the presence of patches and arcs of enhanced electron density within the polar cap ionosphere, Radio Sci., 38 (3), 1052, doi:10.1029/2002RS002798. 